\title{
Alkoholintoxikation - Das sollten Sie wissen für die Ergänzungsprüfung
}

Rico Kuhnke, Thomas Ahne

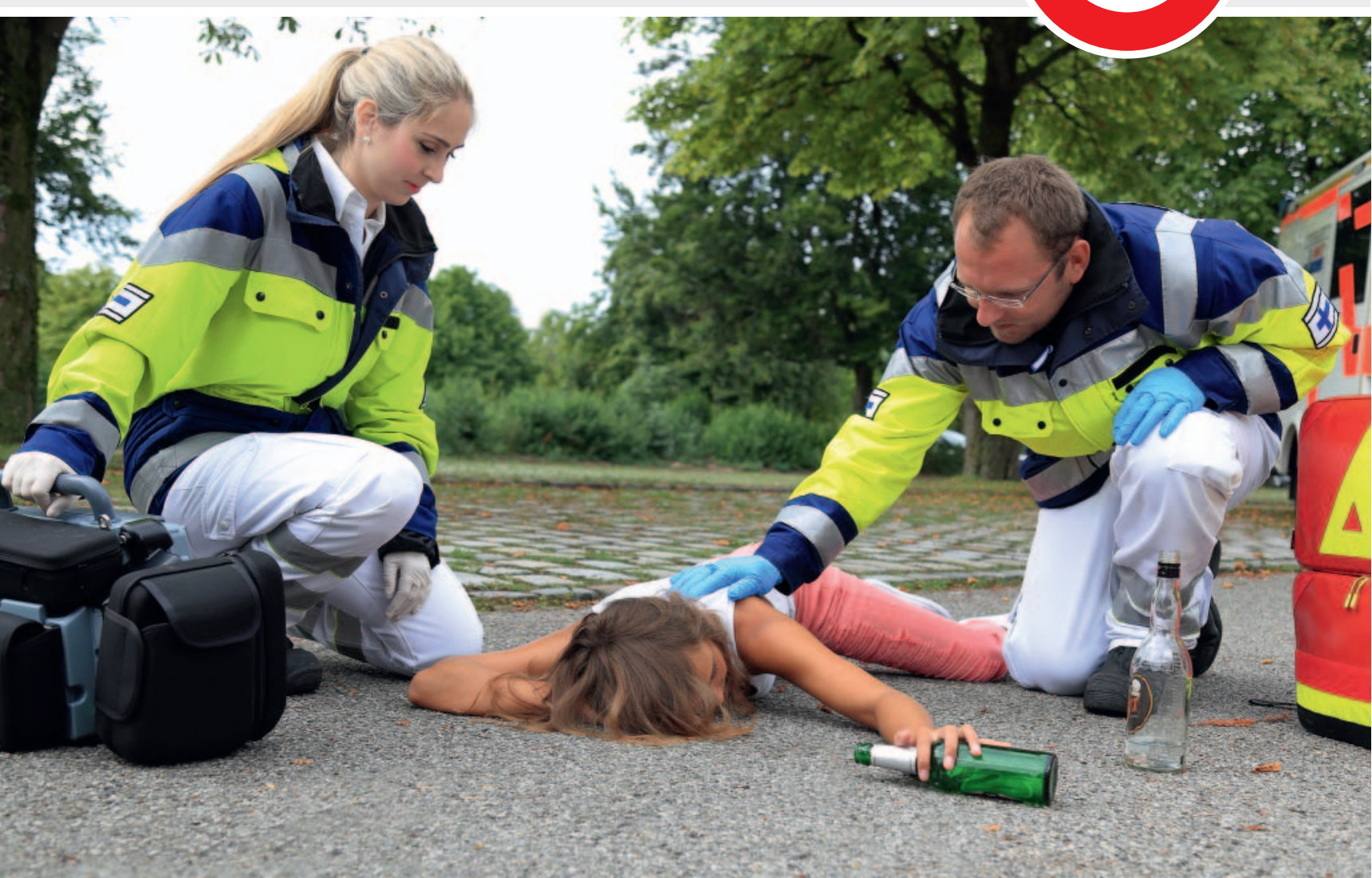

Quelle: RioPatuca Images/stock.adobe.com (nachgestellte Situation)

retten! macht Sie fit für den Notfallsanitäter: In jeder Ausgabe erarbeiten wir anhand eines Fallbeispiels einen interessanten Einsatz algorithmenkonform auf. Anhand von exemplarischen Fragen zu erweiterten Notfallmaßnahmen, Kommunikation und Rahmenbedingungen können Sie sich auf die Ergänzungsprüfung vorbereiten - egal, in welchem Bundesland Sie arbeiten.

\section{Fallbeispiel}

\section{Einsatzmeldung}

Es ist kurz nach Mitternacht im August, als der Rettungsdienst zu einem Notfall in einer Schrebergartensiedlung gerufen wird. Die Leitstelle meldet eine bewusstlose Person mit Verdacht auf Alkoholintoxikation.

An der Einfahrt zur Siedlung wird das Team bereits von einer Gruppe Jugendlicher erwartet. Ein junger Mann bietet an, den Rettungswagen mit seinem Fahrrad zum Notfall- ort zu führen. Am Eingang zu einem Schrebergartengrundstück hält er an und führt die Kollegen in ein kleines Gartenhaus.

\section{Situation vor Ort}

Auf dem Sofa liegt ein 15-jähriges Mädchen in Seitenlage und wird von ihrer Freundin betreut. Die Platz- und Lichtverhältnisse im Gartenhaus sind nicht optimal.

Während sich das Team einen ersten Eindruck verschafft, erzählt die Freundin, sie hätten gemeinsam mit ihren Freunden eine Geburtstagsparty gefeiert. Anna, ihre 
Freundin, sei erst gegen 20 Uhr gekommen und habe dann sehr schnell mehrere Wodka-Energy getrunken. Zuerst habe sie noch ausgelassen getanzt, gegen $23 \mathrm{Uhr}$ sei es ihr schlecht geworden und sie habe sich auf einer Gartenliege ausgeruht.

Kurz vor Mitternacht und dem geplanten Feuerwerk habe sie dann noch einmal nach Anna geschaut. Diese sei nicht mehr ansprechbar gewesen. Beim Transport auf das Sofa im Gartenhaus sei sie kurz wach geworden und habe sich in einen Eimer erbrochen.

\section{Ersteinschätzung}

Die Kollegin bringt die junge Patientin vorsichtig in Rückenlage und überstreckt den Kopf. Die Patientin ist augenscheinlich vigilanzgemindert, sie reagiert weder auf Ansprache noch auf die Lageveränderung. Nur auf einen Schmerzreiz hin verzieht sie stöhnend das Gesicht. Die Atemwege sind frei und der Brustkorb hebt und senkt sich regelmäßig. Der Puls an der A. radialis lässt sich gut tasten. Die ausgezählte Frequenz ist mit 140 Schlägen pro Minute deutlich erhöht, aber regelmäßig.

\section{INTUBATION - JA ODER NEIN?}

Die Glasgow-Coma-Scale (GCS) wurde für die Bewertung von Schädel-Hirn-Traumen (SHT) entwickelt. Daher ist sie nicht 1:1 auf Alkoholintoxikationen anzuwenden. Sie stellt aber dennoch eine gute Richtschnur für die Entscheidung dar, ob eine Schutzintubation zur Atemwegssicherung bei reduzierten Schutzreflexen erfolgen sollte.

Die Atemwegssicherung im Rahmen einer Alkoholintoxikation kann leidenschaftlich diskutiert werden. Einerseits werden die Patienteninnen und Patienten zumeist zügig wieder wacher, andererseits drohen Erbrechen und konsekutive Aspiration. Jede Alkoholintoxikation sicherheitshalber zu intubieren würde jedoch die Möglichkeiten der notärztlichen Versorgung und die Klinikinfrastruktur in den meisten Regionen sprengen.

\section{Transport in den Rettungswagen}

Aufgrund der beengten Verhältnisse entscheidet das Team sich für eine weitere Versorgung im Rettungswagen. Während sich der Kollege aufmacht, den Transport ins Fahrzeug zu organisieren, kümmert sich die Notfallsanitäterin weiter um die junge Patientin.

Diese wird wieder in die Seitenlage gelegt und erhält 5 I Sauersoff über eine Nasenbrille. Zur Überwachung bekommt sie einen Fingerclip. Die Sauerstoffsättigung ist mit $96 \%$ in Ordnung, die Pulsfrequenz bleibt unverän- dert tachykard. Zusätzlich wird eine Absaugpumpe in Bereitschaft gestellt.

Weil die Patientin nicht ansprechbar ist, führt die Kollegin ein Fremdanamnese-Gespräch mit deren bester Freundin. Sie orientiert sich dabei am SAMPLER+S-Schema.

\section{ZUSATZINFO}

Gängige Merkhilfen und Akronyme, vom ABCDESchema über das SAMPLER+S-Schema bis zu den $4 \mathrm{Hs}$ und HITS, haben wir für Sie übersichtlich zusammengefasst. Bestellen Sie Ihren kostenfreien Lernspicker zur Vorbereitung auf Ihre Ergänzungsprüfung unter www.thieme.de/retten-lernspicker.

\section{Fremdanamnese nach SAMPLER+S-Schema}

S-ymptome

Die 15-jährige Anna ist nach wie vor bewusstlos, die gemessenen Vitalparameter sind bis auf die Tachykardie unauffällig.

\section{A-llergien}

Es sind keine Allergien bekannt.

\section{M-edikamente}

Seit etwa drei Monaten nehme Sie regelmäßig die Antibabypille, ansonsten weiß die Freundin von keinen Medikamenten.

\section{P-atientengeschichte}

Von Vorerkrankungen weiß die Freundin nichts. Sie berichtet jedoch, Annas Freund habe gestern unerwartet Schluss gemacht und Anna sei am Boden zerstört - wahrscheinlich habe sie auch deswegen so viel getrunken. Sonst trinke ihre Freundin eigentlich nie Alkohol.

L-etzte Mahlzeit, ...

Bei der Party habe Anna nichts gegessen. Auch wisse sie, dass Anna derzeit eine Diät mache und wahrscheinlich den ganzen Tag noch nichts Richtiges gegessen habe.

\section{E-reignis}

Die Freundin erzählt noch einmal den Verlauf des Abends. Die Kollegin lässt sich den Eimer mit dem Erbrochenen bringen. Wie vermutet, ist die erbrochene Flüssigkeit sehr dünn, Speisereste sind nicht sichtbar, es finden sich keine ungewöhnlichen Hinweise. Das Erbrochene riecht stark süßlich, was sich auf den Energydrink zurückführen lässt. 


\section{R-isikofaktoren}

Keine bekannt.

\section{S-chwangerschaft}

Auf die Frage nach einer Schwangerschaft schüttelt die Freundin den Kopf. Anna nehme ja regelmäßig die Antibabypille, außerdem habe sie in der vergangenen Woche wegen ihrer starken Regelblutung nicht am Sportunterricht teilnehmen können.

\section{0-für-10-Prinzip}

(Quelle: CRM-Leitsätze nach Rall \& Gaba in Miller's Anesthesia 7th edition)

Mittlerweile ist der Kollege mit der Fahrtrage eingetroffen und stellt diese vor der Gartenlaube ab. Das Team nimmt sich kurz Zeit und die Notfallsanitäterin informiert ihren Kollegen über die mittlerweile erhobenen Untersuchungsergebnisse. Gemeinsam stimmen sie das weitere Vorgehen ab.

Nach dem Transport in den Rettungswagen möchte das Team noch einmal eine umfassende Untersuchung vornehmen. Die junge Patientin wird auf ein Tragetuch gerollt und auf die Fahrtrage verbracht.

\section{ABCDE-Schema}

Im Fahrzeug nutzt das Team das C-ABCDE-Schema und vervollständigt die Diagnose. Die Patientin wird mit leicht erhöhtem Oberkörper in Rückenlage gelagert.

A-irway

Die Atemwege sind frei.

B-reathing

Die Atmung ist regelmäßig und mit einer Frequenz von 20 Atemzügen pro Minute unauffällig. Das Pulsoxymeter zeigt nach der Sauerstoffgabe über die Nasensonde einen $\mathrm{SpO}_{2}$ von $97 \%$. Der Brustkorb hebt und senkt sich seitengleich. Beidseits ist ein vesikuläres Atemgeräusch zu hören.

\section{C-irculation}

Nach wie vor lässt sich der Puls an der A. radialis gut tasten. Die Frequenz ist unverändert bei 140 Schlägen pro Minuten. Das mittlerweile angelegte EKG ist, wie zu erwarten, in allen Ableitungen unauffällig. Die Rekapillarisierungszeit des Nagelbetts liegt unter 2 Sekunden. Die Hände der Betroffenen fühlen sich kühl an. Der Blutdruck ist mit $110 / 70 \mathrm{~mm} \mathrm{Hg}$ unauffällig.

\section{D-isability + E-xposure}

Das Team entscheidet, die Patientin bis auf Büstenhalter und Slip zu entkleiden. Bei der Untersuchung geht es strukturiert von kranial nach kaudal vor.

Während der Untersuchung wird die Patientin etwas wacher. Sie gibt inhaltlich inadäquate Laute von sich und zeigt träge, aber gezielte Abwehrmechanismen.

Am Kopf lassen sich keine Verletzungen ausmachen. Die Pupillen sind isokor und reagieren prompt auf Licht. An den Ohren lässt sich kein Blutaustritt feststellen. Der Mundraum ist frei. Ein Zungenbiss lässt sich nicht ausmachen. Die Schluckreflexe sind vorhanden.

Mittlerweile hat der Kollege einen venösen Zugang etabliert und den Blutzucker gemessen. Dieser ist mit $70 \mathrm{mg} / \mathrm{dl}$ zwar etwas niedrig, aber nicht behandlungsbedürftig.

Im HWS-Bereich lässt sich weder Stufenbildung noch Hartspann tasten. Beide Jugularvenen sind unauffällig, der Kehlkopf sitzt mittig. Der Brustkorb ist stabil, das Abdomen weich. Sichtbare Verletzungen sind am Körperstamm nicht feststellbar. Becken, Beine und Arme weisen keine Verletzungszeichen auf. Die Kollegin findet mehrere oberflächliche Schnittwunden am linken Handgelenk.

Die mit einem Ohrthermometer gemessene Körpertemperatur ist mit $35^{\circ} \mathrm{C}$ hypotherm. Die Patientin wird gut zugedeckt und die Heizung im Patientenraum eingeschaltet.

\section{Transport}

Vor dem Transport fasst das Team die Ergebnisse noch einmal zusammen. Aufgrund des Alters von 15 Jahren wird die Patientin in der Kinderklinik des nahe gelegenen Schwerpunktkrankenhauses angemeldet. Der Transport erfolgt in Rückenlage mit leicht erhöhtem Oberkörper und bereitstehender Absaugpumpe und Nierenschale.

\section{Der Algorithmus}

\section{C-ABCDE-Schema}

Im Rettungsdienst gehören Alkoholvergiftungen mit Abstand zu den häufigsten Vergiftungen. Gerade die Häufigkeit der Einsätze birgt die Gefahr, bei der Diagnose mögliche Differenzialdiagnosen nicht ausreichend zu beachten und Dinge zu übersehen. Deshalb ist es von großem Vorteil, routinemäßig alle Punkte des C-ABCDESchemas konsequent abzuarbeiten (siehe $\mathbf{A b b}$. 1).

Unser Team im beschriebenen Fall reduziert sich beim Eintreffen an der Einsatzstelle auf eine orientierende Ersteinschätzung (general impression) und verzichtet auf eine umfangreiche Untersuchung. Es entscheidet 


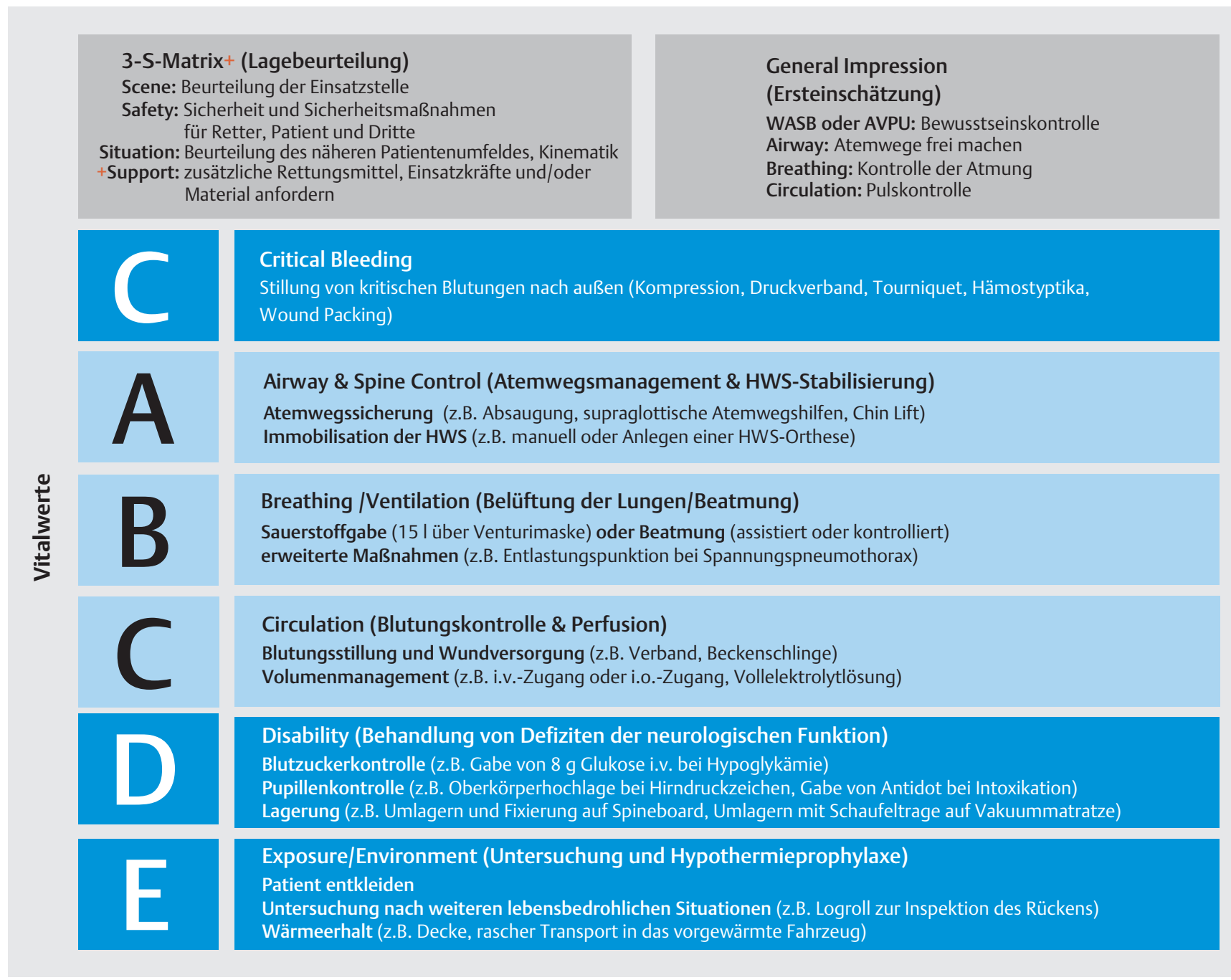

- Abb. 1 C-ABCDE-Schema.

sich, die Patientin zuerst ins Fahrzeug zu verbringen. Die Entscheidung ist aufgrund der beengten Platzverhältnisse in der Gartenlaube gut nachzuvollziehen.

Die gemessenen Vitalparameter machen keine erweiterten Maßnahmen notwendig. Die Patientin wird zum Aspirationsschutz in die Seitenlage gelegt und erhält 5 I Sauerstoff über eine Nasenbrille. Die Gabe von Sauerstoff über eine Nasenbrille kann durchaus kritisch diskutiert werden. Bei der Verdachtsdiagnose Alkoholintoxikation und einer Sauerstoffsättigung von $96 \%$ besteht dafür keine zwingende Notwendigkeit. Viel wichtiger in diesem Zusammenhang ist die Bereitstellung der Absaugeinheit.

\section{Merke}

Tipp: Da die Patienten meist schwallartig erbrechen und die Konsistenz des Erbrochenen häufig dickflüssig ist, kann es hilfreich sein, zuerst ohne aufgesetzten Absaugkatheter, direkt mit dem Absaugschlauch ohne Fingerdip, abzusaugen.

\section{Fremdanamnese}

Weil die Patientin nicht ansprechbar ist, erfolgt die Anamnese mithilfe des SAMPLER+S-Schemas als Fremdanamnese mit der Freundin. Die Ergebnisse sollten allerdings immer kritisch betrachtet werden. Nicht immer haben Freunde und Freundinnen alle Informationen zum Gesundheitszustand der Patientinnen und Patienten - auch nicht die (vermeintlich) besten Freunde. Außerdem ist nie auszuschließen, dass die Möglichkeit besteht, mit falschen Informationen Straftaten zu verschleiern oder sich an der Patientin/dem Patienten zu rächen.

\section{Untersuchung von kranial nach kaudal}

Nach dem Transport ins Fahrzeug nutzt das Team wieder das C-ABCDE-Schema. Das konsequente Abarbeiten des C-ABCDE-Schemas ist sinnvoll. Einziger Nachteil ist, dass verschiedene Körperbereiche im Rahmen der Untersuchung zu unterschiedlichen Zeiten mehrmals untersucht werden müssen. Ein typisches Beispiel ist die Untersuchung des Thorax im Rahmen von B-reathing und 
E-nvironment. Unter B werden die Atembewegungen des Thorax auf Seitengleichheit geprüft, später unter E wird die Stabilität des Thorax geprüft. Dieses Vorgehen birgt insbesondere bei bewusstlosen Patienten die Gefahr, Untersuchungsschritte zu vergessen.

Merke

Tipp: Beginnen Sie mit einer Ersteinschätzung. Prüfen Sie grob die Vitalwerte. Wie ist das Bewusstsein? Nutzen Sie das vereinfachte WASB-Schema $(\mathrm{W}=$ wach, $A=$ auf Ansprache, $S$ = reagiert auf Schmerzreiz, $B=$ bewusstlos). Sind die Atemwege frei? Wie steht es um die Atmung? Prüfen Sie, ob die Atmung ausreichend ist. Wie ist es um den Kreislauf bestellt? Tasten Sie den Puls. Erfordern die Vitalwerte keine sofortige Intervention und ist Ihr Patient bewusstlos, untersuchen Sie Ihren Patienten gründlich von kranial nach kaudal

( $>$ Abb. 2, $\triangleright$ Tab. 1).

\section{Untersuchungsergebnisse}

Bis auf die unbedeutenden Schnittwunden und eine leichte Unterkühlung scheint sich die Diagnose Alkoholintoxikation bei der jungen Patientin zu bestätigen. Auch wenn die Schnittwunden nur oberflächlich sind zusammen mit den Erzählungen der besten Freundin ist anzunehmen, dass sich unsere Patientin aufgrund der Trennung von ihrem Freund in einer Lebenskrise befindet. Damit diese Informationen nicht verloren gehen, sollten sie in jedem Fall auf dem Protokoll vermerkt werden.

\section{Prüfungsfragen}

\section{NOTFALLMEDIZIN}

? Erläutern Sie, wie sich Alkoholkonsum auswirkt und welche Stadien ein Patient mit zunehmendem Konsum durchläuft.

Alkohol wirkt direkt auf das zentrale Nervensystem. Die Resorptionsgeschwindigkeit nach oraler Aufnahme schwankt stark und ist von der Magenfüllung abhängig. In Abhängigkeit von der Blutalkoholkonzentration können vier Stadien einer Alkoholvergiftung unterschieden werden.

Die nachfolgende Einteilung dient nur als grobe Orientierung. Die Übergänge sind fließend und dynamisch. Insbesondere bei Patienten, bei denen ein Gewöhnungseffekt an Alkohol eingetreten ist, lassen die Symptome keine Rückschlüsse auf die tatsächliche Alkoholkonzentration zu.

\section{Stadium 1}

$(0,5-1,5 \%)$ Exzitatorisches Stadium

Die Patienten sind häufig euphorisiert und redselig. Die Sprache wirkt verwaschen. Oft sind die Patienten zunehmend distanzlos, enthemmt oder reizbar. Es treten erste Gangunsicherheiten und Koordinationsstörungen auf.

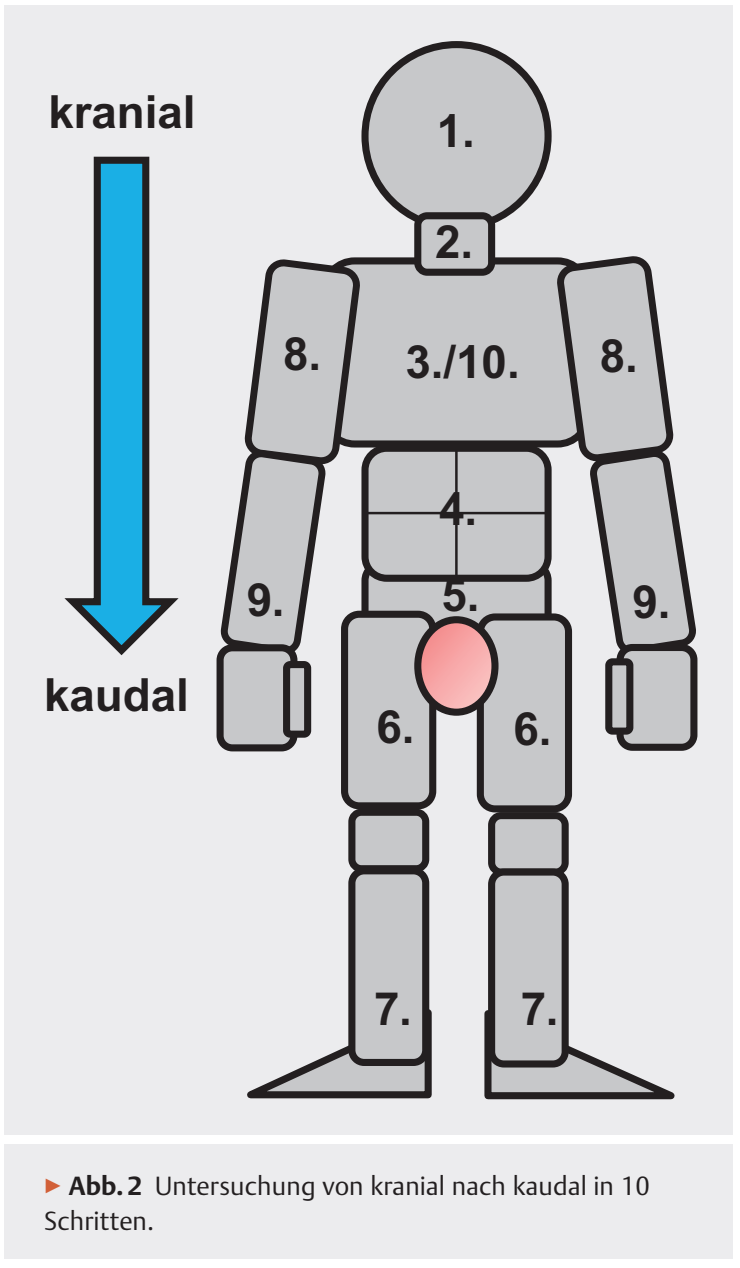

\section{Stadium 2}

$(1,5-2,5 \%$ ) Hypnotisches Stadium Die Patienten werden zunehmend ruhig. Gangunsicherheiten und Koordinationsstörungen werden massiver. Es treten schwere Sprechstörungen auf (Lallen).

\section{Stadium 3}

$(2,5-3,5 \%)$ Narkotisches Stadium

Die Patienten sind zunehmend verwirrt, die Bewusstseinsstörungen reichen von einer Somnolenz bis zum Koma. Häufig tritt im Zusammenhang mit dem Abbau des Alkohols eine Hypoglykämie auf.

\section{Stadium 4}

(ab 3,5\% $\%_{0}$ ) Asphyktisches Stadium Tiefes Koma, keine Reaktion auf Schmerzreize, Erlöschen der Schutzreflexe. Zunehmende Atmungs- und Kreislaufprobleme bis hin zum Herzkreislaufstillstand. 
- Tab. 1 Untersuchung von kranial nach kaudal in 10 Schritten.

1. Kopf

*, Stabilität der Schädelknochen prüfen, nach Verhärtungen, Stufenbildung und Schmerzäußerungen im Bereich der HWS suchen, Pupillenkontrolle (z. B. Seitendifferenz bei SHT, Miosis bei Opiatintoxikation, Mydriasis bei Kokain- oder Amphetaminintoxikation, träge Lichtreaktion bei Benzodiazepinabusus, gerötete Bindehaut bei THC-Konsum, Einblutungen bei Erdrosselungen, Gelbfärbung der Bindehaut bei Hepatitis ...), Lidschlussreflex prüfen, Mundinspektion (z. B. Schwellung bei allergischer Reaktion, Zungenbiss bei Krampfanfall, Himbeerzunge bei Scharlach ...), Ausatemgeruch = Foetor ex ore (z. B. Azetongeruch bei Hyperglykämie, Uringeruch bei Nierenausfallkoma), Ohrinspektion (z. B. Blutung bei Schädelbasisfraktur), Temperaturmessung (z. B. Fieber bei Infektionen, Unterkühlung)

2. Hals *, nach Verhärtungen und/oder Schmerzäußerungen im Nackenbereich suchen, Stufenbildung HWS tasten, Status Jugularvenen (z. B. Jugularvenenstauung bei Herzinsuffizienz oder Spannungspneumothorax), Lagekontrolle Kehlkopf (z. B. Verschiebung bei Spannungspneumothorax)

3. Thorax

*, Stabilität des Thorax prüfen, Atemexkursionen beobachten (z. B. einseitig bei Pneumothorax, reduziert bei flacher Atmung oder Bronchoobstruktion), Auskultation Lunge (z. B. einseitig bei Pneumothorax, inspiratorische Atemnebengeräusche bei Bolusgeschehen, endexspiratorisches Pfeifen oder Giemen bei Brochoobstruktion bzw. Silent Chest, Brodeln bei Lungenödem ...), Auskultation Herz (z. B. zunehmend gedämpft bei Herzbeuteltamponade, Strömungsgeräusche bei Klappenschlussproblemen ...), Perkussion (z. B. Schachteltongeräusch bei Pneumothorax)

\begin{tabular}{|l|l|}
\hline 4. Abdomen & $\begin{array}{l}\text { *, Tasten der } 4 \text { Quadranten (z. B. Abwehrspannung bei Einblutungen, Loslassschmerz oder Rovsing- } \\
\text { Symptom bei Blinddarmentzündung ...), Auskultation Magen und Darm (z. B. keine Darmgeräusche } \\
\text { bei paralytischem lleus, hochgestellte Darmgeräusche als Hinweis auf mechanischen lleus ...) }\end{array}$ \\
\hline 5. Becken & ${ }^{*}$, Stabiliät des Beckenknochens prüfen (z. B. Open-Book-Phänomen bei Beckenfraktur) \\
\hline 6. Oberschenkel & *, Stabilität der Oberschenkenknochen prüfen \\
\hline 7. Unterschenkel und Füße & $\begin{array}{l}\text { *, Stabilität der Unterschenkelknochen prüfen, auf Fehlstellungen achten (z. B. verkürzt nach außen } \\
\text { rotiert bei Oberschenkelhalsfraktur), Prüfen der Beweglichkeit }\end{array}$ \\
\hline 8. Oberarme & *, Stabilität der Oberarmknochen prüfen \\
\hline 9. Unterarme und Hände & *, Stabilität der Unterarmknochen prüfen, Nagelbettprobe (z. B. verzögert als Anzeichen für Schock) \\
\hline 10. Rücken & *, nach Stufenbildung und Schmerzäußerungen im Wirbelsäulenbereich suchen \\
\hline ggf. Genitalbereich & $\begin{array}{l}\text { Achtung, Intimsphäre beachten! } \\
\text { Nur bei konkretem Verdacht auf Verletzungen, bei bewusstseinsklaren Patienten vor einer Inspektion } \\
\text { immer zuerst Zustimmung einholen und Maßnahme im Vorfeld begründen und erläutern }\end{array}$ \\
\hline
\end{tabular}

* Suche nach sichtbaren Verletzungen und Hautveränderungen: Wunden (Schnitt-, Biss- und Platzwunden, Hautabschürfungen, Verbrennungen), Prellmarken, Hautrötungen, Ekzeme, Schwellungen, Ödembildungen, Einstichstellen ...

\section{Mögliche weitere Fragen:}

- Wie kann sich die Kombination von koffeinhaltigen Getränken mit Alkohol auswirken?

- Beschreiben Sie eine gründliche Untersuchung von kranial nach kaudal. Nennen Sie dabei mögliche pathologische Befunde und die dazugehörige Diagnose.

\section{KOMMUNIKATION}

? Was versteht man unter Closing-Loop-Strategie?

Unter Closing-Loop-Strategie versteht man, dass der Empfänger einer Information diese dem Sender kurz spiegelt bzw. kurz wiederholt. Gerade im Einsatz kann durch diese Strategie verhindert werden, dass Informationen nicht oder falsch verstanden werden. Die Rückkopplung schafft im Einsatz die notwendige Sicherheit zwischen den Einsatzkräften.

\section{Mögliche weitere Fragen:}

- Beschreiben Sie mögliche Ängste der Freundin von Anna und reflektieren Sie deren Bedürfnisse.

\section{RAHMENBEDINGUNGEN}

? Ein stark alkoholisierter Patient mit einer Kopfplatzwunde verweigert den Transport. Beurteilen Sie die rechtliche Situation und beschreiben Sie, wie Sie sich in dieser Situation verhalten würden.

Aufgrund des starken Alkoholkonsums ist der Patient nicht oder nur eingeschränkt geschäftsfähig bzw. einwilligungsfähig. Er ist nicht in der Lage, die Konsequenzen seiner Entscheidung umfassend zu beurteilen.

Ein Transport in die Klinik gegen den Willen des Patienten muss sich am geltenden Unterbringungsgesetz des Landes orientieren. Grundsätzlich ist die Polizei befugt, bei einer Eigen- oder Fremdgefährdung des Patienten eine „Zwangseinweisung“ vorzunehmen. 
Im konkreten Fall kann man versuchen, dem Patienten die Notwendigkeit eines Transports zu erläutern und auf das Hinzuziehen der Polizei zu verweisen. Hält der Patient die Transportverweigerung aufrecht, ist die Polizei zum Einsatz hinzuzuziehen. Das Akzeptieren einer Transportverweigerung bei Einwilligungsunfähigkeit des Patienten kann zu straf- und zivilrechtlichen Konsequenzen führen.

\section{Mögliche weitere Fragen:}

- Bewerten Sie für das oben beschriebene Fallbeispiel, ob die Eltern der jungen Anna umgehend zu informieren sind.

- Muss die Polizei verständigt werden?

- Ist die Entscheidung, die 15-jährige Patienten in die Kinderklinik zu bringen, richtig? Begründen Sie Ihre Antwort.

\section{KOMMENTAR}

von Dr. med. Thomas Ahne, Facharzt für Anästhesiologie mit Zusatzbezeichnung Notfallmedizin

Patienten mit einer Alkoholintoxikation gehören zum Alltag im deutschen Rettungsdienst. Grundsätzlich muss man unterscheiden, ob es sich um einen Notfall mit einem alkoholisierten Patienten bei bekannter Alkoholkrankheit oder um eine akute Vergiftung ohne vorbestehenden chronischen Alkoholabusus handelt, da dies unterschiedliche Ursachen haben kann, die dann auch andere Maßnahmen nach sich ziehen.

Mahnend muss man sich vor Augen halten, dass alkoholisierte Patienten oft qualitativ schlechter behandelt werden (prä- wie innerklinisch) als nicht alkoholisierte. Man muss sich bemühen, diese Patienten mit gleich hohem Anspruch und unvoreingenommen zu versorgen, sonst entsteht schnell eine deutlich erhöhte Rate an Fixierungsfehlern, die für den Patienten eine Gefährdung darstellen können. Alkoholabhängigkeit ist eine Volkskrankheit. Der medizinisch betrachtet schädliche Konsum größerer Mengen ist mehrheitlich öffentlich akzeptiert und toleriert. Unsere Gesellschaft leistet sich (auch finanziell) dieses legale Rauschmittel. Oft steht nicht der (von mir persönlich geteilte) Genuss am Getränk, sondern schlicht die Zufuhr von Ethanol im Vordergrund. Einerseits wird seit Jahren in Deutschland insgesamt immer weniger Alkohol konsumiert, andererseits gibt es besorgniserregende Entwicklungen wie den hohen Konsum junger Menschen (oft auch Minderjähriger), insbesondere von Mischgetränken bzw. Alkopops.
Es scheint mir lohnenswert, dass jeder in der Notfallmedizin Tätige sich mit diesem Thema auseinandersetzt und sich eine fundierte Meinung dazu bildet, denn die Konfrontation damit ist Alltag - und dann ist in der konkreten Situation ein besonnenes und professionelles Auftreten und Handeln gefragt.

\section{Interessenkonflikt}

Die Autoren geben an, dass kein Interessenkonflikt besteht.

Autorinnen/Autoren

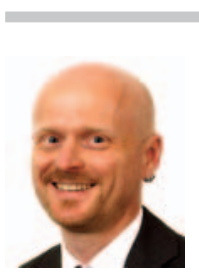

\section{Rico Kuhnke}

MA. Gesamtschulleiter der DRK-Landesschule Baden-Württemberg. Er war viele Jahre als Lehrrettungsassistent tätig und hat sein pädagogisches Masterstudium berufsbegleitend abgeschlossen. Er ist Notfallsanitäter und Mitherausgeber von retten!

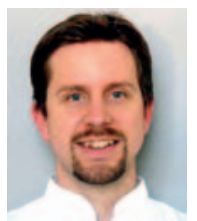

\section{Thomas Ahne}

Dr. med. Facharzt für Anästhesiologie mit Zusatzbezeichnung Notfallmedizin. Der Mitherausgeber von retten! macht derzeit einen Quereinstieg in die Allgemeinmedizin am Gesundheitszentrum Todtnau. Präklinisch ist er, boden- wie luftgebunden, in Deutschland, Österreich und der Schweiz tätig.

Korrespondenzadresse

Rico Kuhnke

Schulleiter

Deutsches Rotes Kreuz

Landesschule Baden-Württemberg

Karl-Berner-Str. 6

72295 Pfalzgrafenweiler

r.kuhnke@drk-Is.de

\section{Bibliografie}

DOI https://doi.org/10.1055/a-0865-8357

retten 2019; 8: 244-250

(c) Georg Thieme Verlag KG, Stuttgart · New York ISSN 2193-2387 